\title{
Risk of hip fracture in patients on dialysis or kidney transplant: a meta-analysis of 14 cohort studies
}

This article was published in the following Dove Press journal:

Therapeutics and Clinical Risk Management

\section{Jiachang $\operatorname{Tan}^{1, *}$ \\ Yuping $\mathrm{Li}^{2}, *$ \\ Zhenjie $\mathrm{Wu}^{1, *}$ \\ Jinmin Zhao ${ }^{3}$}

'Department of Bone and Soft Tissue Surgery, Affiliated Tumor Hospital, Guangxi Medical University, Nanning 53002 I, People's Republic of China; ${ }^{2}$ The First Clinical Medical College, Guangxi Medical University, Nanning 53002 I, People's Republic of China; ${ }^{3}$ Department of Orthopaedics Trauma and Hand Surgery, The First Affiliated Hospital, Guangxi Medical University, Nanning 53002I, People's Republic of China

*These authors contributed equally to this work
Correspondence: Jinmin Zhao Department of Orthopaedics Trauma and Hand Surgery, The First Affiliated Hospital, Guangxi Medical University, Shuangyong Rd. \#6, Nanning, Guangxi 53002 I, People's Republic of China

Tel: +86-77I-53525 I2

Fax: +86-77I-53525I2

Email gxzj1962@I63.com
Purpose: We aimed to conduct a meta-analysis of published cohort studies to evaluate the risk of hip fracture in patients undergoing dialysis or kidney transplantation (KT).

Methods: We identified relevant studies by searching PubMed, EMBASE and Google Scholar databases from their inception to December 31, 2017. Cohort studies evaluating risk of hip fractures in patients undergoing dialysis or KT were considered included. The methodological quality of the cohort studies was assessed using the modified Newcastle-Ottawa scale.

Results: In our meta-analysis of 14 retrospective cohort studies, a total of more than 1.5 million patients undergoing dialysis or KT were included, of whom more than 30,000 had hip fractures. After the merger, the proportion of hip fractures was $1.92 \%$ (95\% CI, 1.38\%-2.46\%) with significant heterogeneity $\left(I^{2}=99.9 \%, P=0.000\right)$ in all patients, and the incidence rate of hip fractures (per 1,000 person-years) was 8.95 (95\% CI, 4.05-13.85) with significant heterogeneity $\left(I^{2}=99.9 \%\right.$, $P=0.000)$. The pooled relative risks (RR) value for dialysis patients compared with the general population were 6.35 (95\% CI, 4.53-8.88) for male and 5.57 (95\% CI, 4.44-6.99) for female. The pooled RR value for hemodialysis (HD) patients compared with peritoneal dialysis (PD) patients was $1.39(95 \% \mathrm{CI}, 1.13-1.70)$ with no heterogeneity $\left(I^{2}=0.0 \%, P=0.763\right)$.

Conclusion: In conclusion, the present meta-analysis reveals that about $2 \%$ of dialysis or KT patients go on to sustain a hip fracture during follow-up, with the overall hip fracture incidence rates being 8.95 per 1,000 person-years. The overall risk of hip fracture was more than 5 -fold higher in dialysis patients than in the general population. Among patients on PD, HD, and KT, HD and KT patients had the highest and the lowest risk of hip fractures, respectively.

Keywords: risk, hip fracture, dialysis or kidney transplant, meta-analysis

\section{Introduction}

Hip fracture is a major concern among the general population. Such factures result in extensive morbidity and mortality, and they impose a severe economic burden. ${ }^{1,2}$ One study has predicted that the number of hip fractures in the world will increase from 1.66 million in 1990 to 6.26 million by $2050 .^{3}$

Recently, some studies have shown that patients undergoing dialysis or kidney transplant (KT) have a higher risk of hip fracture as compared to the general population. ${ }^{4-6}$ The incidence of hip fracture in dialysis or KT patients is between 1.9 and 29.3 events per 1,000 person-years. ${ }^{4-16}$ A population-based cohort study has found that the total mortality rate in dialysis patients with hip fractures is more than twice that of dialysis patients without such fractures. ${ }^{8}$ Dialysis patients with hip fractures have been shown to have a standardized mortality rate 2.4 times greater than that of those in the general population with hip fractures..$^{5}$ Therefore, greater attention is needed when treating patients undergoing dialysis or KT, such as the use of osteoporosis 
drugs or other formulated measures aimed at reducing the risk of hip fracture in these at-risk individuals.

However, some studies have reported inconsistent findings with regard to the risk of hip fracture in patients with dialysis or KT. To address such inconsistencies, we therefore performed a meta-analysis using data from cohort studies to assess the risk of hip fracture in dialysis or KT patients.

\section{Materials and methods}

\section{Search strategy}

The present meta-analysis was conducted based on the Preferred Reporting Items for Systematic Reviews and Meta-Analyses (PRISMA) statement. ${ }^{17}$ Two reviewers (JT and YL) independently searched PubMed, EMBASE, and Google Scholar from their inception to December 31, 2017 using a combination of Medical Subject Heading (MeSH), free terms, and relevant keywords. The PubMed search strategy was as follows: (Transplantation [MeSH] OR "Kidney Transplantation" [MeSH] OR "Transplantation, Heterotopic" [MeSH] OR "Renal Dialysis" [MeSH]) AND ("Hip Fractures" [MeSH]). And the search strategy for free terms was as follows: (Transplantation OR Transplantations OR Transplant OR Transplants OR Dialysis OR Dialyses) AND (Hip OR Hips) AND (Fractures OR Fracture). The reference lists of all the selected articles were hand searched for further studies.

\section{Selection criteria}

The inclusion criteria for this meta-analysis were as follows: 1) studies were cohort (both prospective and retrospective) studies; 2) hip fractures were the fracture type; 3) incidence of hip fracture in patients undergoing dialysis or KT was reported; 4) data were not repeated in other articles. If studies did not meet these criteria, they were excluded. The selection of studies was conducted independently by two reviewers. Inconsistencies in selection were resolved by a third reviewer.

\section{Data extraction}

Two reviewers (JT and YL) independently read the selected studies and extracted the appropriate data. The data extracted included first author's name, study year, country or region, study period, number of dialysis or KT patients, type of patients (HD, PD, and KT), patients' age, proportion of males, follow-up durations, follow-up (person-years), number of hip fractures, proportion of hip fractures (\%), and incident rate of hip fractures (per 1,000 person-years).

\section{Quality assessment}

The methodological quality of included cohort studies was assessed using the modified Newcastle-Ottawa scale, which consists of three factors: patient selection, comparability of the study groups, and assessment of outcome. ${ }^{18}$ A score of 0-9 (given as stars) was allocated to each study. Studies scoring 0-3, 4-6, and 7-9 were considered low-, medium-, and high-quality studies, respectively.

\section{Statistical analysis}

The incidence rate of hip fracture (per 1,000 person-years) in patients undergoing dialysis or KT were extracted from the original data, and the proportions of hip fracture (\%) were the result of dividing the total number of hip fractures by the total number of patients undergoing dialysis or KT. To evaluate the relative risk (RR) and corresponding 95\% CI of hip fracture among those on dialysis as compared to the general population and in HD as compared to PD, we combined the adjusted RRs and 95\% CIs reported in the original studies. A sensitivity analysis was also carried out to eliminate each study, one at a time, from the meta-analysis. Results from a random-effects model were reported in the present meta-analysis. A heterogeneity assessment between the included studies was based on $I^{2}$ statistics. The $I^{2}$ values were $25 \%, 50 \%$ and $75 \%$ respectively, indicating low, medium and high degree of heterogeneity. The Begg's test and Egger's test were used to assess the publication bias. All statistical analyses were conducted using STATA version 11.0. $P$-values were two-sided, and $P<0.05$ was defined as being statistically significant.

\section{Results \\ Search results}

The specific literature selection steps employed in this study are shown in Figure 1. From the three reviewed databases (PubMed, EMBASE, and Google Scholar) and other sources, a total of 2,534 studies were retrieved. After removing duplicates, a total of 752 studies remained. Then 595 studies were excluded after reviewing titles and abstracts. After a full text review of the remaining 157 studies, 143 articles were excluded, including 24 reviews, 26 case studies, and 58 studies that did not have specific data on hip fractures, and 35 studies that lacked available data. Finally, a total of 14 retrospective cohort studies ${ }^{4-7,9-14,19-22}$ were included in the present meta-analysis.

\section{Study characteristics and quality}

The characteristics of the 14 included studies are shown in Table 1. In this meta-analysis, a total of more than 1.5 million 
2,509 identified citations screened in PubMed + Google Scholar + EMBASE

25 additional citations identified through other sources

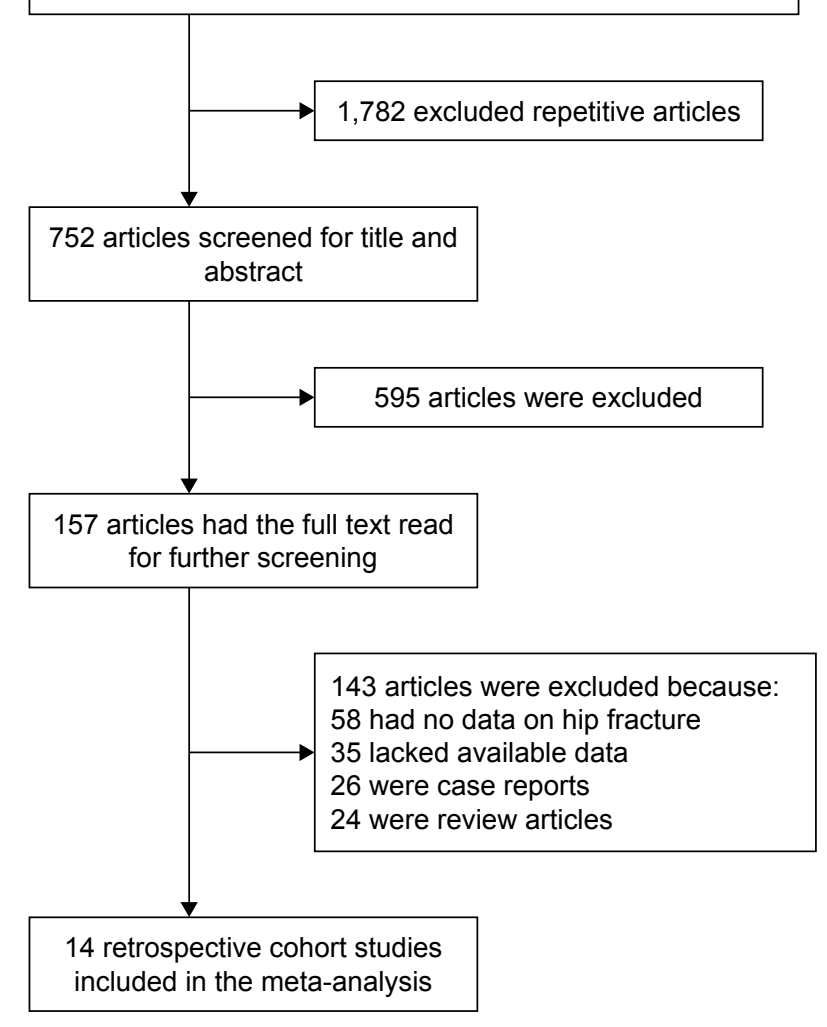

Figure I The steps of the literature selection.

patients undergoing dialysis or KT were included, of whom more than 30,000 had hip fractures. Among these 14 retrospective cohort studies, the year of publication ranged from $2000^{4,5,19}$ to $2016 .^{22}$ Eight studies were conducted in the United States, ${ }^{4,5,7,9-11,19,20}$ two in Japan, ${ }^{6,14}$ two in Taiwan, ${ }^{12,13}$ one in Canada $^{22}$ and one in England. ${ }^{21}$ The age of dialysis or KT patients ranged from $47^{7}$ to $76^{11}$ years, and sample sizes ranged from $1,272^{5}$ to $409,040^{11}$ patients, and average follow-up durations were between $1^{6,10,14}$ and $10^{22}$ years. The proportions of hip fracture in dialysis or KT patients ranged from $0.84 \% \%^{12,19}$ to $4.40 \%,{ }^{5}$ and the incidence rate was $1.54^{21}$ to 16.6 per 1,000 person-years. ${ }^{10}$ Twelve studies ${ }^{4,6-14,20-22}$ were considered to be of high methodologic quality, and only two studies ${ }^{7,19}$ were of moderate quality (Table 1 ).

\section{Proportions and incidence rate of hip fractures in patients undergoing dialysis or $\mathrm{KT}$}

Of the 14 included studies, seven studies ${ }^{6,9,10,12-14,19}$ included a total of 645,418 patients on HD, of whom 10,791 had hip frac-

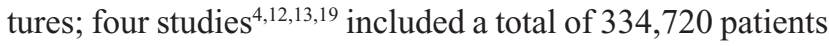
on $\mathrm{PD}$, of whom 6,642 had hip fractures; three studies ${ }^{5,7,11}$ included 451,407 HD and PD patients of whom 22,559 had hip fractures, and five studies ${ }^{7,19-22}$ included a total of 154,316 KT patients, of whom 1,362 had hip fractures. After pooling these data, the overall incidence of hip fractures (Figure 2) was $2.28 \%(95 \% \mathrm{CI}, 1.63 \%-2.93 \%)$ with significant heterogeneity $\left(I^{2}=99.8 \%, P=0.000\right)$ in HD patients, $1.46 \%(95 \%$ CI, $0.70 \%-2.23 \%)$ with significant heterogeneity $\left(I^{2}=96.1 \%\right.$, $P=0.000)$ in PD patients, $3.25 \%(95 \%$ CI, $0.56 \%-5.94 \%)$ with significant heterogeneity $\left(I^{2}=99.9 \%, P=0.000\right)$ in $\mathrm{PD}$ and HD patients, $0.89 \%$ (95\% CI, $0.78 \%-1.00 \%)$ with moderate heterogeneity $\left(I^{2}=69.4 \%, P=0.011\right)$ in KT patients, and $1.92 \%(95 \%$ CI, $1.38 \%-2.46 \%)$ with significant heterogeneity $\left(I^{2}=99.9 \%, P=0.000\right)$ in all patients. The incidence rate of hip fractures (per 1,000 person-years, Figure 3) was 11.70 (95\% CI, 9.44\%-13.96\%) with significant heterogeneity $\left(I^{2}=91.5 \%, P=0.000\right)$ in HD patients, $7.60(95 \%$ CI, 3.54$11.65)$ with significant heterogeneity $\left(I^{2}=54.3 \%, P=0.087\right)$ in PD patients, 15.40 (95\% CI, -5.66-36.46) with significant heterogeneity $\left(I^{2}=99.8 \%, P=0.000\right)$ in PD and HD patients, 2.98 (95\% CI, 2.08-3.88) with no heterogeneity $\left(I^{2}=0.0 \%\right.$, $P=0.420)$ in KT patients, and $8.95(95 \% \mathrm{CI}, 4.05-13.85)$ with significant heterogeneity $\left(I^{2}=99.9 \%, P=0.000\right)$ in all patients. The individual removal of specific studies one at a time did not significantly alter the overall results. No evidence of publication bias was found when evaluated using Begg's test $(P=0.26)$, or Egger's test $(P=0.15)$.

\section{RR of hip fracture in patients on dialysis relative to the general population}

The RR of hip fracture in patients on dialysis is greater than that of the general population. Three of the included studies $^{4,5,16}$ reported this comparison. The pooled RR value was 6.35 (95\% CI, 4.53-8.88) for males and 5.57 (95\% CI, 4.44-6.99) for females (Figure 4).

\section{RR of hip fractures in HD patients relative to PD patients}

Three of the included studies ${ }^{12,13,19}$ reported the RR value for HD patients compared with PD patients. The pooled RR value was 1.39 (95\% CI, 1.13-1.70) with no heterogeneity $\left(I^{2}=0.0 \%, P=0.763\right)$ (Figure 5).

\section{Discussion}

The present meta-analysis of 14 retrospective cohort studies including a total of more than 1.5 million patients undergoing dialysis or KT and more than 30,000 hip fracture patients reveals that about $2 \%$ of dialysis or KT patients had sustained a hip fracture during follow-up, with the overall hip fracture incidence rates being 8.95 per 1,000 person-years. 


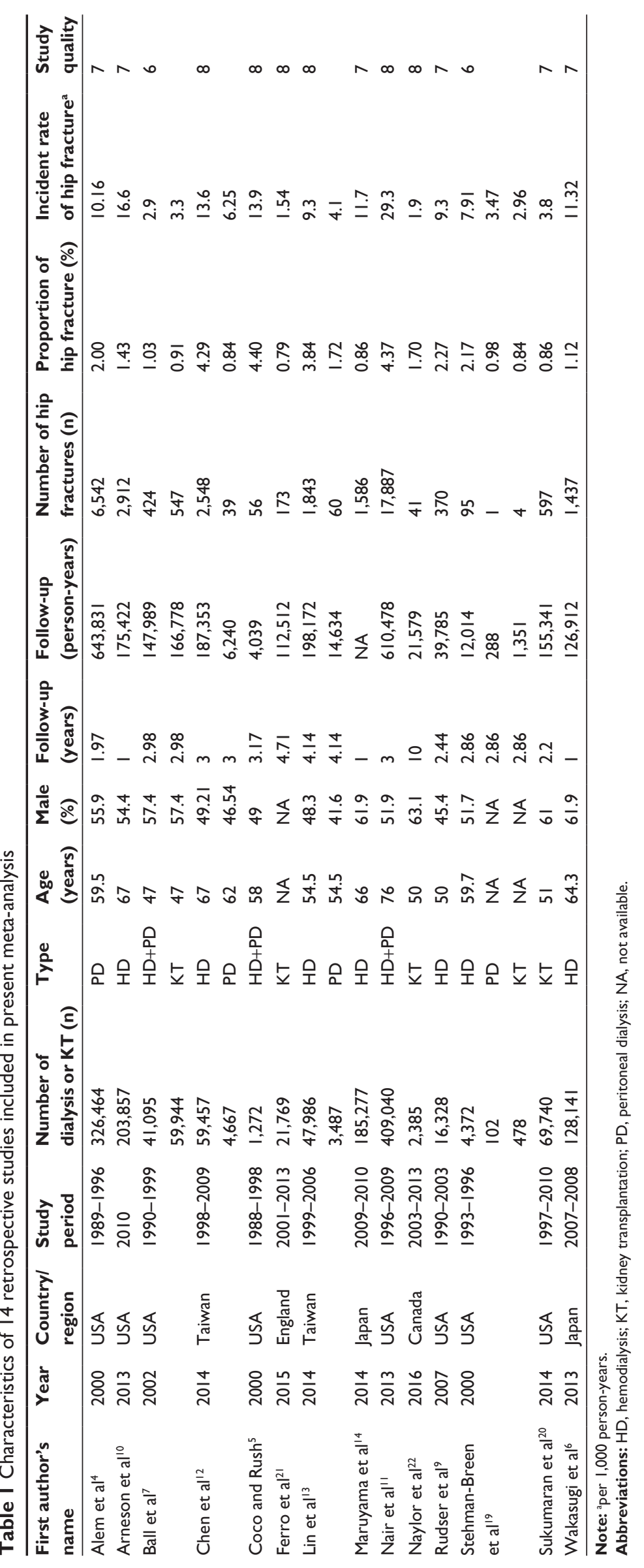




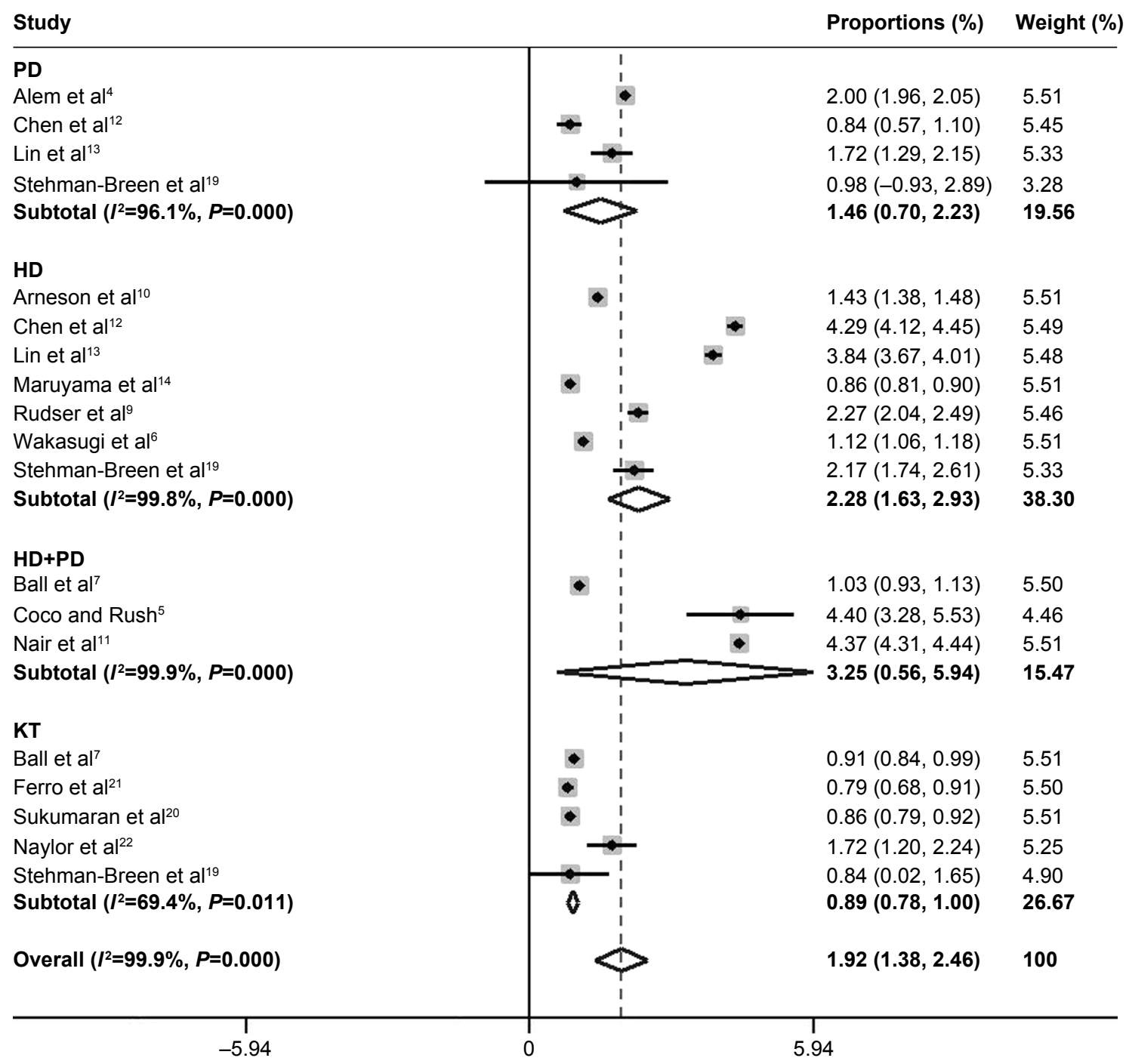

Figure 2 Proportions (\%) of hip fracture in patients on dialysis or KT. Note: Weights are from random effects analysis.

Abbreviations: $\mathrm{HD}$, hemodialysis; $\mathrm{KT}$, kidney transplantation; $\mathrm{PD}$, peritoneal dialysis.

The overall RR of hip fracture was more than 5-fold higher in dialysis patients than in the general population. Furthermore, the RR of hip fracture was higher in patients on HD, who had a 1.39-fold increase in their risk of suffering a hip fracture as compared to patients on PD.

The high incidence of hip fracture in patients on dialysis or KT has the potential to substantially impact patient health and standards of living. Dialysis patients with hip fractures had a 2.4-fold greater standardized mortality rate than those in the general population with hip fractures. ${ }^{5}$ The mechanisms governing this high risk of hip fracture among patients undergoing dialysis or KT are not well understood, and are likely related to multiple comorbidities in such patients, including secondary hyperparathyroidism, abnormal vitamin D metabolism, hypogonadism, chronic acidosis, and related disorders. ${ }^{23,24} \mathrm{~A}$ major driver of this elevated fracture rate is likely the lower bone mineral density (BMD) in these patients. ${ }^{25}$ Low BMD is itself an independent risk factor for hip fracture in the general population ${ }^{26}$ and has been associated with fractures in patients undergoing dialysis ${ }^{27}$ and $\mathrm{KT}^{2}{ }^{28}$

Among patients undergoing PD, HD, and KT, those undergoing HD and KT had the highest and the lowest risk of hip fractures in our meta-analysis, respectively. Different dialysis methods are associated with different risk rates for hip fracture. The risk of hip fracture in patients on HD was 1.39-fold higher than that in patients on PD in our metaanalysis. One possible explanation for this finding may be that patients on PD were on average younger than those on $\mathrm{HD}$, whereas patients on HD had a higher incidence rate of diabetes than did those patients on PD. ${ }^{29}$ Although we found no data directly comparing the incidence of hip fractures 


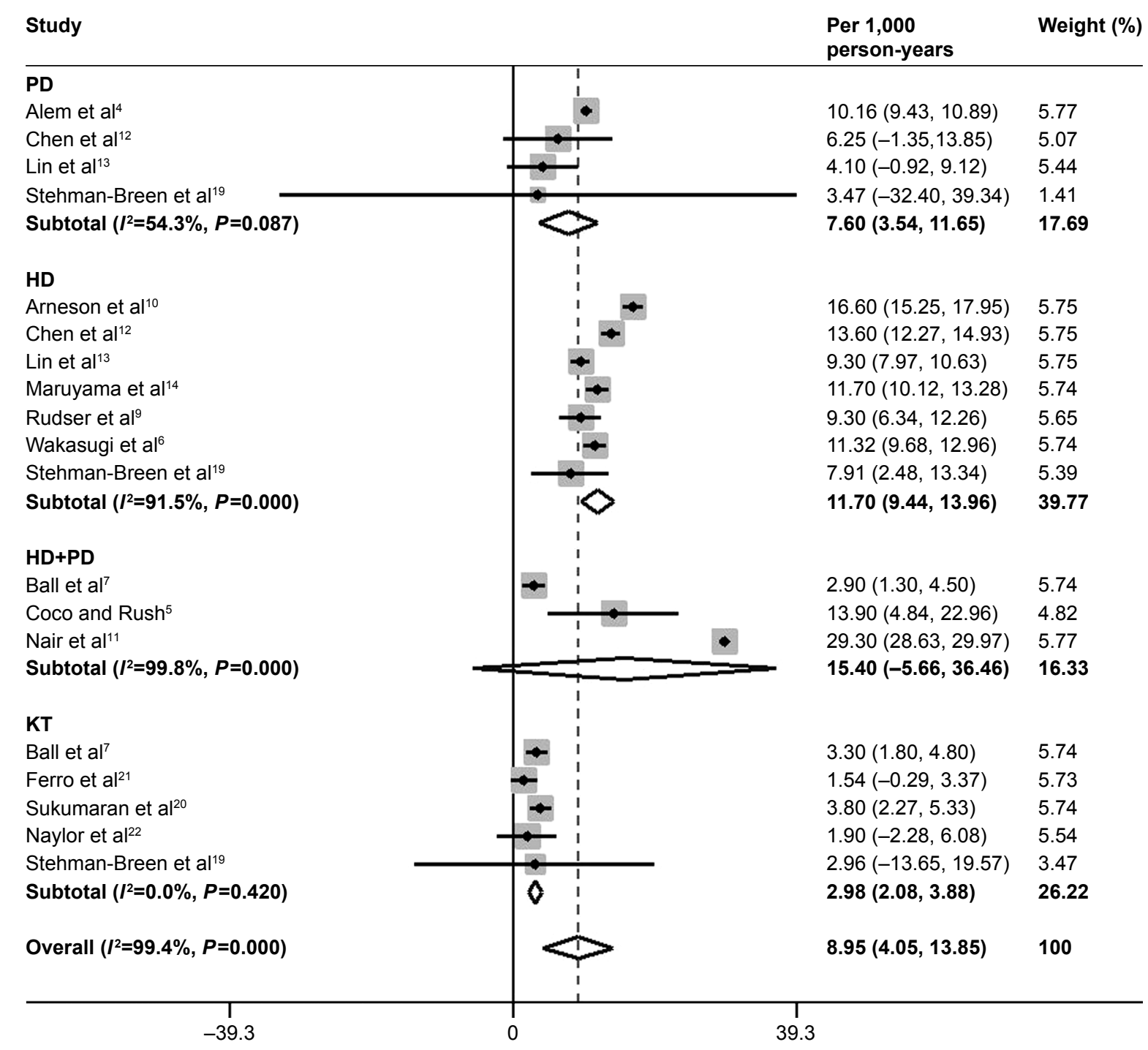

Figure 3 Incidence rate of hip fracture (per I,000 person-years) in patients on dialysis or KT.

Note: Weights are from random effects analysis.

Abbreviations: HD, hemodialysis; KT, kidney transplantation; PD, peritoneal dialysis.

between patients undergoing dialysis and $\mathrm{KT}$, data from this meta-analysis (Figures 1 and 2) indicate that the proportions and incidence rate of hip fractures in patients on KT were significantly lower than those of patients on dialysis. Certainly, further research will be needed to fully validate this finding. Based on these results, however, greater attention should be paid to dialysis patients, especially those on HD, by offering them osteoporosis therapy, improved health education, fall prevention strategies, and other techniques aimed at reducing their risk of hip fracture.

This meta-analysis does have several limitations. For one, the 14 studies included in this meta-analysis were all retrospective cohort studies based on historical data. Unlike prospective cohort studies, the quality of these retrospective cohort studies was not controlled by the researchers, so if there were other confounding factors then the reliability and integrity of the study was likely to be low, affecting the quality and reliability of our meta-analysis. However, all meta-analyses are limited by such constraints on the quality and quantity of published studies, and they remain the best approach to resolving such problems at present, serving as a basis for decision making by clinicians and patients. Secondly, the present meta-analysis revealed high heterogeneity between studies, which was not explained by any one of the included studies and which may reduce the overall reliability of this study. This high heterogeneity may be associated with differences in age, gender, sample size, or follow-up time between the included studies. Caution is therefore required in the interpretation of our conclusions. Thirdly, the current meta-analysis lacks a pre-registered review protocol and there may be small biases, but we strictly follow the systematic evaluation process (PRISMA). Finally, we were not able to 


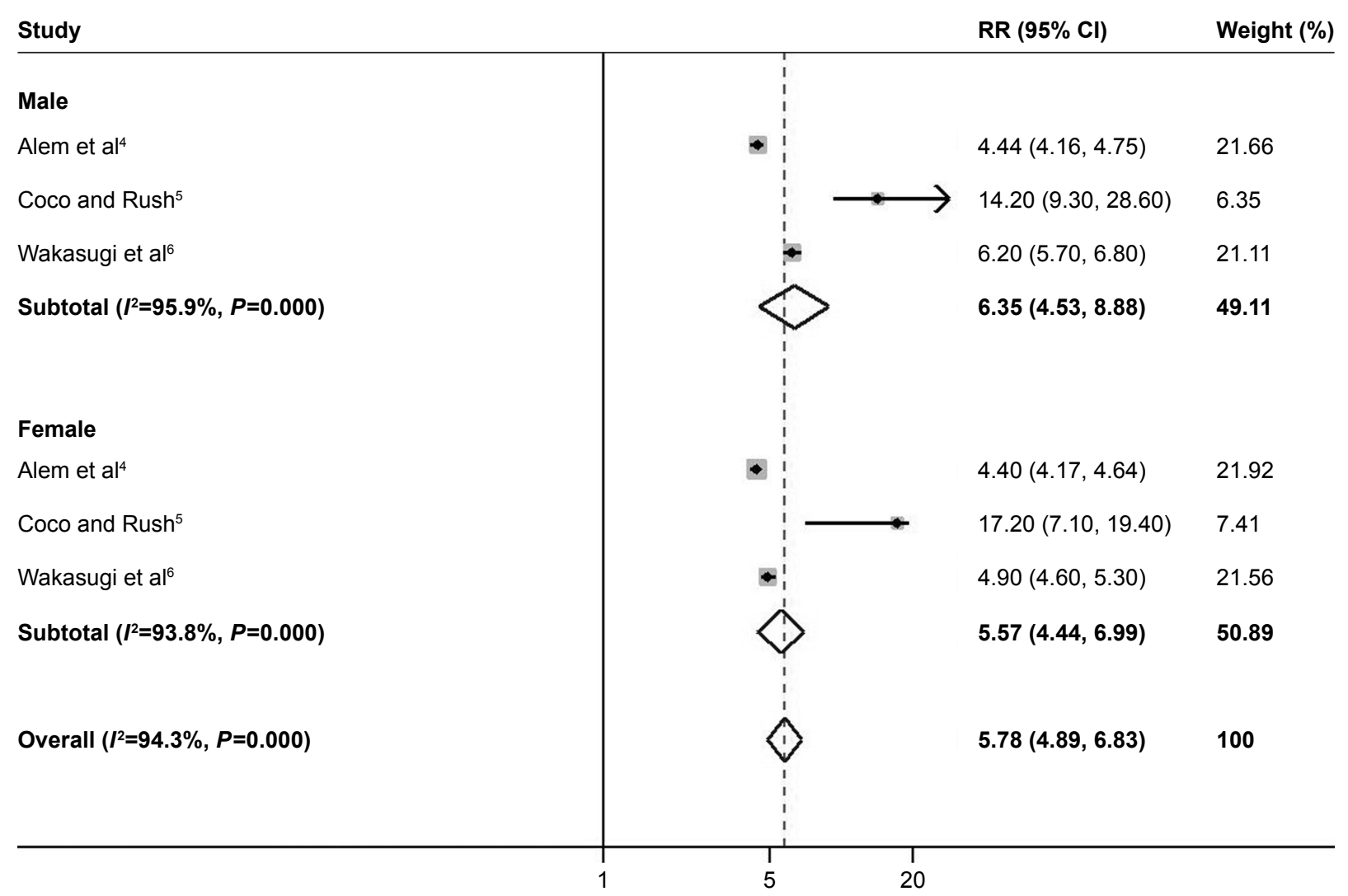

Figure 4 RR of hip fracture in patients on dialysis compared to the general population. Note: Weights are from random effects analysis.

Abbreviation: RR, relative risk.

include any unpublished data in this analysis, and so we may have overlooked some research that is eligible for publication. However, the problem is unlikely to be significant because the Egger and Begg's tests indicated that there was no publication bias in the current meta-analysis. Although our meta-analysis is subject to these limitations, it also has certain advantages. For one, this is a comprehensive literature review, and it is the first to our knowledge to analyze the risk of hip fracture

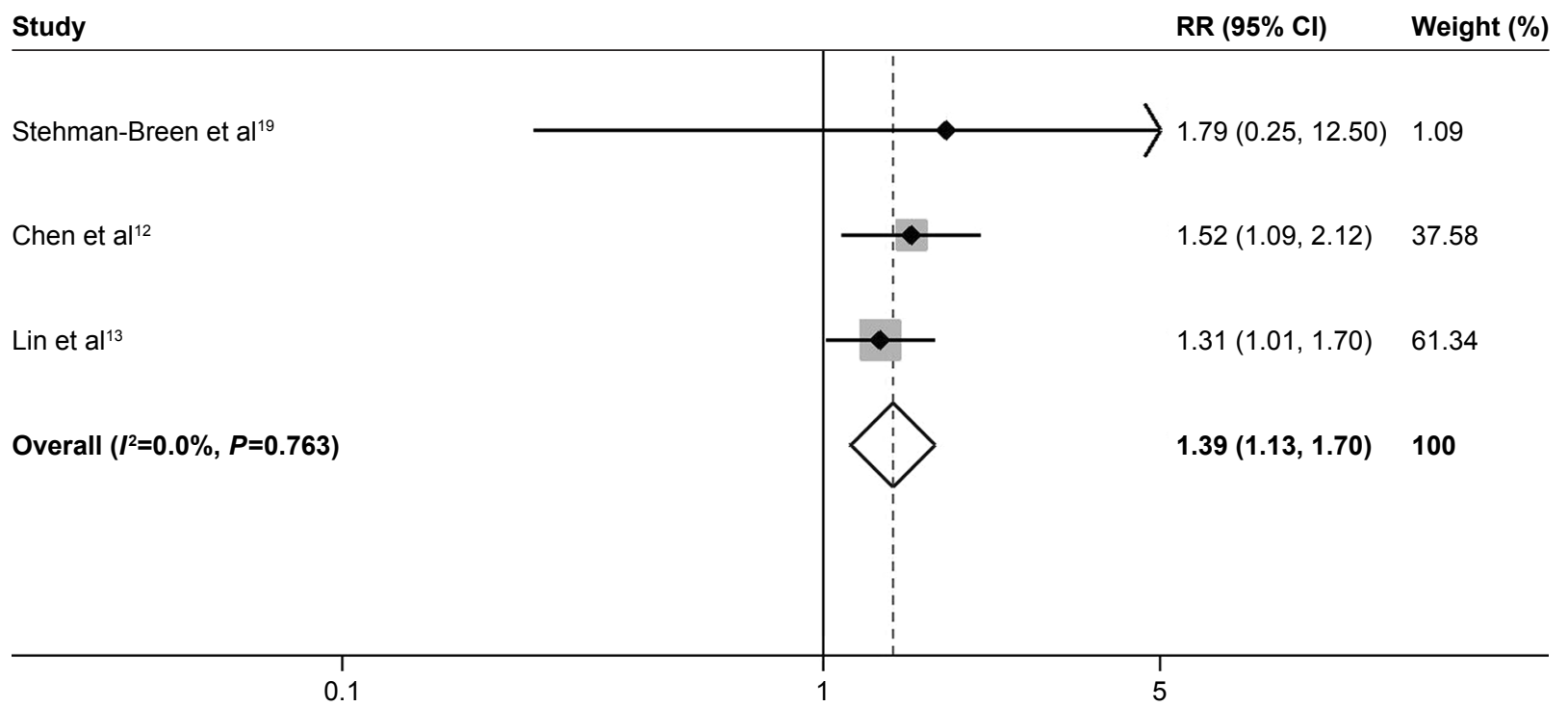

Figure 5 RR of hip fractures in patients on HD compared to those on PD. 
after dialysis or KT. In addition, in the present meta-analysis, many cohort studies included a large number of dialysis or KT patients and had long-term follow-up durations, greatly enhancing the statistical power of the combined results.

\section{Conclusion}

The present meta-analysis showed that about $2 \%$ of dialysis or KT patients go on to sustain a hip fracture during follow-up, with the overall hip fracture incidence rates being 8.95 per 1,000 person-years. The overall risk of hip fracture was more than 5-fold higher in dialysis patients than in the general population. Among patients on PD, HD, and KT, HD patients and KT patients had the highest and the lowest risk of hip fractures, respectively.

\section{Author contributions}

JT and JZ were involved with the concept and design of this manuscript. JT, YL and ZW were involved with the data collection, analysis, and writing of the paper. All authors contributed toward drafting and critically revising the paper, gave final approval of the version to be published, and agree to be accountable for all aspects of the work. All authors contributed toward data analysis, drafting and critically revising the paper and agree to be accountable for all aspects of the work.

\section{Disclosure}

The authors report no conflicts of interest in this work.

\section{References}

1. Burge R, Dawson-Hughes B, Solomon DH, Wong JB, King A, Tosteson A. Incidence and economic burden of osteoporosis-related fractures in the United States, 2005-2025. J Bone Miner Res. 2007; 22(3):465-475.

2. Braithwaite RS, Col NF, Wong JB. Estimating hip fracture morbidity, mortality and costs. J Am Geriatr Soc. 2003;51(3):364-370.

3. Cooper C, Campion G, Melton LJ. Hip fractures in the elderly: a worldwide projection. Osteoporos Int. 1992;2(6):285-289.

4. Alem AM, Sherrard DJ, Gillen DL, et al. Increased risk of hip fracture among patients with end-stage renal disease. Kidney Int. 2000; 58(1):396-399.

5. Coco M, Rush $\mathrm{H}$. Increased incidence of hip fractures in dialysis patients with low serum parathyroid hormone. Am J Kidney Dis. 2000;36(6): 1115-1121.

6. Wakasugi M, Kazama JJ, Taniguchi M, et al. Increased risk of hip fracture among Japanese hemodialysis patients. J Bone Miner Metab. 2013; 31(3):315-321.

7. Ball AM, Gillen DL, Sherrard D, et al. Risk of hip fracture among dialysis and renal transplant recipients. JAMA. 2002;288(23):3014-3018.

8. Mittalhenkle A, Gillen DL, Stehman-Breen CO. Increased risk of mortality associated with hip fracture in the dialysis population. Am J Kidney Dis. 2004;44(4):672-679.

9. Rudser KD, de Boer IH, Dooley A, Young B, Kestenbaum B. Fracture risk after parathyroidectomy among chronic hemodialysis patients. J Am Soc Nephrol. 2007;18(8):2401-2407.
10. Arneson TJ, Li S, Liu J, Kilpatrick RD, Newsome BB, St Peter WL. Trends in hip fracture rates in US hemodialysis patients, 1993-2010. Am J Kidney Dis. 2013;62(4):747-754.

11. Nair SS, Mitani AA, Goldstein BA, Chertow GM, Lowenberg DW, Winkelmayer WC. Temporal trends in the incidence, treatment, and outcomes of hip fracture in older patients initiating dialysis in the United States. Clin J Am Soc Nephrol. 2013;8(8):1336-1342.

12. Chen YJ, Kung PT, Wang YH, et al. Greater risk of hip fracture in hemodialysis than in peritoneal dialysis. Osteoporos Int. 2014;25(5): 1513-1518.

13. Lin ZZ, Wang JJ, Chung CR, et al. Epidemiology and mortality of hip fracture among patients on dialysis: Taiwan National Cohort Study. Bone. 2014;64:235-239.

14. Maruyama Y, Taniguchi M, Kazama JJ, et al. A higher serum alkaline phosphatase is associated with the incidence of hip fracture and mortality among patients receiving hemodialysis in Japan. Nephrol Dial Transplant. 2014;29(8):1532-1538.

15. Mathew AT, Hazzan A, Jhaveri KD, et al. Increasing hip fractures in patients receiving hemodialysis and peritoneal dialysis. Am J Nephrol. 2014;40(5):451-457.

16. Wakasugi M, Kazama JJ, Wada A, et al. Regional variation in hip fracture incidence among Japanese hemodialysis patients. Ther Apher Dial. 2014;18(2):162-166.

17. Moher D, Liberati A, Tetzlaff J, Altman DG; PRISMA Group. Preferred reporting items for systematic reviews and meta-analyses: the PRISMA statement. Ann Intern Med. 2009;151(4):264-269.

18. Stang A. Critical evaluation of the Newcastle-Ottawa scale for the assessment of the quality of nonrandomized studies in meta-analyses. Eur J Epidemiol. 2010;25(9):603-605.

19. Stehman-Breen CO, Sherrard DJ, Alem AM, et al. Risk factors for hip fracture among patients with end-stage renal disease. Kidney Int. 2000;58(5):2200-2205.

20. Sukumaran Nair S, Lenihan CR, Montez-Rath ME, et al. Temporal trends in the incidence, treatment and outcomes of hip fracture after first kidney transplantation in the United States. Am J Transplant. 2014;14(4):943-951.

21. Ferro CJ, Arnold J, Bagnall D, Ray D, Sharif A. Fracture risk and mortality post-kidney transplantation. Clin Transplant. 2015;29(11): 1004-1012.

22. Naylor KL, Jamal SA, Zou G, et al. Fracture Incidence in Adult Kidney Transplant Recipients. Transplantation. 2016;100(1):167-175.

23. Delgado C, Frassetto L. Hip fractures in hemodialysis patients. Am J Kidney Dis. 2013;62 (4):653-655.

24. Leinau L, Perazella MA. Hip fractures in end-stage renal disease patients: incidence, risk factors, and prevention. Semin Dial. 2006; 19(1):75-79.

25. Stein MS, Packham DK, Ebeling PR, Wark JD, Becker GJ. Prevalence and risk factors for osteopenia in dialysis patients. Am J Kidney Dis. 1996;28(4):515-522.

26. Cummings SR, Nevitt MC, Browner WS, et al. Risk factors for hip fracture in white women. Study of Osteoporotic Fractures Research Group. N Engl J Med. 1995;332(12):767-773.

27. Bucur RC, Panjwani DD, Turner L, Rader T, West SL, Jamal SA. Low bone mineral density and fractures in stages 3-5 CKD: an updated systematic review and meta-analysis. Osteoporos Int. 2015;26(2):449-458.

28. Kan SL, Ning GZ, Chen LX, Zhou Y, Sun JC, Feng SQ. Efficacy and safety of bisphosphonates for low bone mineral density after kidney transplantation: a meta-analysis. Medicine. 2016;95(5):e2679.

29. Chien CC, Wang JJ, Sun YM, et al. Long-term survival and predictors for mortality among dialysis patients in an endemic area for chronic liver disease: a national cohort study in Taiwan. BMC Nephrol. 2012; 13:43. 
Therapeutics and Clinical Risk Management

Dovepress

\section{Publish your work in this journal}

Therapeutics and Clinical Risk Management is an international, peerreviewed journal of clinical therapeutics and risk management, focusing on concise rapid reporting of clinical studies in all therapeutic areas, outcomes, safety, and programs for the effective, safe, and sustained use of medicines. This journal is indexed on PubMed Central, CAS,

EMBase, Scopus and the Elsevier Bibliographic databases. The manuscript management system is completely online and includes a very quick and fair peer-review system, which is all easy to use. Visit http://www.dovepress.com/testimonials.php to read real quotes from published authors.

Submit your manuscript here: http://www.dovepress.com/therapeutics-and-clinical-risk-management-journal 